\title{
Lower levels of plasma NT-proBNP are associated with higher prevalence of NASH in patients with biopsy-proven NAFLD
}

\author{
Authors: \\ Zeng-Pei Qiao ${ }^{1 *}$, Kenneth I. Zheng ${ }^{2 *}$, Pei-Wu Zhu ${ }^{3}$, Feng Gao ${ }^{4}$, Hong-Lei Ma ${ }^{2}$, Gang Li ${ }^{2}$, Yang- \\ Yang $\mathrm{Li}^{5}$, Giovanni Targher ${ }^{6}$, Christopher D. Byrne ${ }^{7}$, Ming-Hua Zheng ${ }^{2,8,9 \$}$
}

\section{Affiliations:}

${ }^{1}$ Department of Laboratory Medicine, the Second Affiliated Hospital of Wenzhou Medical University \& Yuying Children's Hospital, Wenzhou, China;

${ }^{2}$ NAFLD Research Center, Department of Hepatology, the First Affiliated Hospital of Wenzhou Medical University, Wenzhou, China;

${ }^{3}$ Department of Clinical Laboratory, the First Affiliated Hospital of Wenzhou Medical University, Wenzhou, China;

${ }^{4}$ Department of Gastroenterology, the First Affiliated Hospital of Wenzhou Medical University, Wenzhou, China;

${ }^{5}$ Department of Pathology, the First Affiliated Hospital of Wenzhou Medical University, Wenzhou, China;

${ }^{6}$ Section of Endocrinology, Diabetes and Metabolism, Department of Medicine, University and Azienda Ospedaliera Universitaria Integrata of Verona, Verona, Italy;

${ }^{7}$ Southampton National Institute for Health Research Biomedical Research Centre, University Hospital Southampton, Southampton General Hospital, Southampton, UK;

${ }^{8}$ Institute of Hepatology, Wenzhou Medical University, Wenzhou, China; 
${ }^{9}$ The Key Laboratory of Diagnosis and Treatment for The Development of Chronic Liver Disease in Zhejiang Province, Wenzhou, China.

*Co-first authors: Zeng-Pei Qiao and Kenneth I. Zheng.

Keywords: N-terminal pro-brain natriuretic peptide; nonalcoholic fatty liver disease; nonalcoholic steatohepatitis; cardiovascular disease; biopsy-proven.

\section{${ }^{\$}$ Corresponding author:}

Ming-Hua Zheng, MD, PhD

NAFLD Research Center, Department of Hepatology, the First Affiliated Hospital of Wenzhou Medical University; No. 2 Fuxue Lane, Wenzhou 325000, China.

E-mail: zhengmh@wmu.edu.cn; fax: (86) 577-55578522; tel: (86) 577-55579622.

\section{List of Abbreviations:}

NT-proBNP, N-terminal pro-brain natriuretic peptide; NAFLD, nonalcoholic fatty liver disease; NASH, nonalcoholic steatohepatitis; HOMA-IR, homeostasis model assessment of insulin resistance. 


\begin{abstract}
Background and Aims. Emerging evidence suggests that plasma N-terminal pro-brain natriuretic peptide (NT-proBNP) levels are decreased in patients with imaging-defined nonalcoholic fatty liver disease (NAFLD), but no data are currently available on the association between plasma NT-proBNP levels and the histologic severity of NAFLD.
\end{abstract}

Methods and Results. We enrolled 351 (73.5\% men) consecutive adult patients with biopsyproven NAFLD without a prior history of cardiovascular disease. Plasma NT-proBNP levels were measured using a commercially available immunochemical system (VITROS ${ }^{\text {. }} 5600$, Johnson, New Jersey). Fifty-three percent of these subjects had nonalcoholic steatohepatitis (NASH). After stratification of patients by plasma NT-proBNP tertiles; compared to those in the $1^{\text {st }}$ tertile (NT-proBNP $\left.\leq 16 \mathrm{pg} / \mathrm{ml}\right)$, the odds ratio for NASH was $0.52(95 \% \mathrm{CI} 0.29-0.95)$ in patients in the $2^{\text {nd }}$ tertile (NT-proBNP of $\left.17-33 \mathrm{pg} / \mathrm{ml}\right)$ and $0.49(95 \%$ CI $0.26-0.93)$ in those in the $3^{\text {rd }}$ tertile (NT-proBNP $\geq 34 \mathrm{pg} / \mathrm{ml}$ ) of plasma NT-proBNP levels, even after adjustment for age, sex, body mass index, HOMA-estimated insulin resistance, pre-existing diabetes, hypertension and dyslipidemia.

Conclusions. In subjects with biopsy-proven NAFLD without known cardiovascular disease, this cross-sectional study shows for the first time, that lower plasma NT-proBNP levels are strongly associated with a higher prevalence of NASH. 


\section{BACKGROUND}

Nonalcoholic fatty liver disease (NAFLD) is defined as excessive accumulation of triglycerides in the liver without other competing causes of hepatic fatty infiltration. Nonalcoholic steatohepatitis (NASH) is the potentially progressive form of NAFLD that also increases the risk of developing cardiovascular disease (CVD). ${ }^{1}$ One of the major causes for development of NAFLD is thought to be a compromised capacity to oxidize excess fatty acids in the liver. ${ }^{2}$ Over time, chronic inflammatory state induced by hepatic steatosis may progress into NASH.

Natriuretic peptides (NP) are encoded in the form of longer inactive peptides, which can be transformed into shorter active NPs and equimolar N-terminal pro-brain natriuretic peptide (NTproBNP) by enzyme corin and furin. ${ }^{3,4}$ NT-proBNP is served as a circulating biomarker for NP production and activation.

Emerging evidence has suggested that plasma levels of NT-proBNP are decreased amongst patients with imaging-defined NAFLD. ${ }^{5,6}$ Since BNP plays an important role in stimulating lipid oxidation and inhibiting visceral fat accumulation ${ }^{7}$, it is plausible that BNP can also play a role in NAFLD development. Since it is currently not known whether there is any association between plasma NT-proBNP levels and the histologic severity of NAFLD, the major aim of this study was to investigate the association between plasma NT-proBNP levels and the histological characteristics of NAFLD. 


\section{METHODS}

\section{Patients}

We enrolled 351 adult individuals with biopsy-proven NAFLD, who did not have a prior history of CVD (defined by self-reported history, plasma NT-proBNP levels $>200 \mathrm{pg} / \mathrm{ml}$, or electrocardiographic changes suggestive of myocardial ischemia or infarction). These patients were consecutively recruited between December 2016 and February 2019 at the First Affiliated Hospital of Wenzhou Medical University, from an investigator initiated study cohort. ${ }^{8}$ Patients enrolled in the cohort were subject to the following eligibility criteria: (1) age between 18-75 years; (2) presence of hepatic steatosis on imaging methods and then confirmed by liver biopsy; and (3) provided informed written consent prior to their inclusion in the study. Subjects were excluded if they met the following criteria: (1) excessive alcohol intake (i.e. $\geq 20$ grams of alcohol per day for the past 2 years); (2) hepatic diseases other than NAFLD, such as viral hepatitis (defined as positivity for hepatitis B virus surface antigen, hepatitis B viral DNA, antihepatitis $\mathrm{C}$ virus antibodies or hepatitis $\mathrm{C}$ virus RNA), cholestatic hepatitis or autoimmune hepatitis (diagnosed by serum autoantibodies and/or histology); (3) overt or subclinical kidney dysfunction, defined as a history of renal disease, overt proteinuria, estimated glomerular filtration rate $<90 \mathrm{ml} / \mathrm{min} / 1.73 \mathrm{~m}^{2}$ or serum creatinine $>133 \mu \mathrm{mol} / \mathrm{l}$; and (4) active cancers.

The study was approved by the local ethics committee of the First Affiliated Hospital of Wenzhou Medical University (2016-246, approved on December 2016). The study protocol conforms to the ethical guidelines of the 1975 Declaration of Helsink. Each subject has provided written informed consent. 


\section{Clinical and biochemical parameters}

All blood samples were obtained from all participants on the same day of liver biopsy, after at least an 8-hour fast. Blood samples were tested by standard methods in our central laboratory. Routine biochemical tests included measurement of high-sensitivity C-reactive protein (hs-CRP), leptin, alanine aminotransferase (ALT), aspartate aminotransferase (AST), alkaline phosphatase, gamma-glutamyl transpeptidase (GGT), albumin, platelet count, prothrombin time, glucose, insulin, hemoglobin A1c, and lipid profile. Plasma NT-proBNP level was measured by using a commercially available immunochemical system (VITROS ${ }^{\circ} 5600$, Johnson, New Jersey).

Height, weight, waist circumference and hip circumference were recorded, in light clothing and without shoes, on the same day of liver biopsy. Body mass index (BMI) was calculated as weight (kg) divided by squared height (m). Diagnoses of diabetes, metabolic syndrome, hypertension, and dyslipidemia were made according to commonly used diagnostic criteria, as previously described. ${ }^{9}$ Homeostasis model assessment of insulin resistance (HOMA-IR) was also calculated by using an established formula. ${ }^{10}$

\section{Liver histology}

All patients underwent ultrasound-guided percutaneous biopsy (16-gauge Hepafix needle), as described previously. ${ }^{11}$ Liver specimens were embedded in paraffin and underwent H\&E and Masson's trichrome staining. Liver histologic assessment was performed by a single experienced liver pathologist. The pathologist scored the samples according to the NASH-Clinical Research Network system, and was blinded to clinical and biochemical data for all participants. ${ }^{12}$ Patients were stratified into two groups: non-NASH and NASH (defined as NAFLD Activity Score 
$[\mathrm{NAS}] \geq 4$ with a score $\geq 1$ point for each of the histological components of steatosis, ballooning and lobular inflammation) ${ }^{12}$.

\section{Statistical analysis}

In order to investigate the association between plasma NT-proBNP levels and the presence of NASH, the participants were stratified by NT-proBNP tertiles. The one-way ANOVA, KruskalWallis and Fisher's exact tests were then used, as appropriate, to examine the differences in patient characteristics across NT-proBNP tertiles. Binary logistic regression analyses adjusted for age, sex, BMI, HOMA-IR score, hypertension, diabetes and dyslipidemia were undertaken to test the independent association between NT-proBNP tertiles and presence of NASH [SPSS Version R23.0.0 (IBM, Armonk, NY)].

\section{RESULTS}

\section{Baseline characteristics}

A total of 351 consecutive patients with biopsy-proven NAFLD were included in this study.

Table 1 shows the clinical and liver histology characteristics of patients stratified by plasma NTproBNP tertiles. The age of participants increased significantly and proportion of male sex decreased as plasma NT-proBNP level increased. There were significant differences in adiposity measures, fasting insulin levels, HOMA-IR score and proportion of both diabetes and hypertension (but not dyslipidemia) among the three groups of patients. In addition, there was a significant difference in serum ALT levels, but not in other liver enzyme levels. Notably, the 
proportion of patients with NASH, as well as the median of the total NAS score decreased significantly across NT-proBNP tertiles. While there were significant inter-group differences in the grade of steatosis and hepatic ballooning across NT-proBNP tertiles, no significant differences were found in lobular inflammation or fibrosis stage (although patients in the $1^{\text {st }} \mathrm{NT}$ proBNP tertile tended to have higher $\mathrm{F} \geq 2$ stages).

\section{Association between plasma NT-proBNP and NASH}

To further investigate the association between plasma NT-proBNP levels and presence of NASH, we performed a logistic regression analysis (Table 2). Compared to patients in the lower NT-proBNP tertile (NT-proBNP $\leq 16 \mathrm{pg} / \mathrm{ml}$ ), those in the $2^{\text {nd }}$ tertile (NT-proBNP of $17-33 \mathrm{pg} / \mathrm{ml}$ ) and those in the $3^{\text {rd }}$ tertile (NT-proBNP $\geq 34 \mathrm{pg} / \mathrm{ml}$ ) had a lower risk of having NASH. In particular, after adjusting for age, sex, BMI, HOMA-IR score, hypertension, dyslipidemia and diabetes (Table 2; adjusted model 2), the odds ratio for NASH was 0.52 (95\% CI 0.29-0.95, $p=0.03)$ in patients in the $2^{\text {nd }}$ tertile and $0.49(95 \%$ CI $0.26-0.93, p=0.03)$ in those in the $3^{\text {rd }}$ tertile of plasma NT-proBNP levels, respectively.

\section{Boxplot analysis of plasma NT-proBNP}

A boxplot of plasma NT-proBNP levels in relation to total NAS score and NASH status was reported in Figure 1. Plasma levels of NT-proBNP decreased progressively in relation to both total NAS and presence of NASH.

\section{DISCUSSION}


We showed that lower plasma NT-proBNP levels are significantly associated with a higher prevalence of NASH in a large cohort of Chinese patients with biopsy-proven NAFLD who did not have a prior history of CVD. This association was independent of age, sex, HOMA-IR score and features of the metabolic syndrome.

Whilst other investigators have previously shown that plasma NT-proBNP levels were significantly decreased in individuals with magnetic resonance imaging or computed tomography-defined NAFLD, ${ }^{5,6}$ however, these studies were unable to investigate the relationship between plasma NT-proBNP levels and risk of having biopsy-confirmed NASH.

Earlier studies have also shown that higher circulating NT-pro BNP levels were significantly associated with lower adipose tissue mass in humans. The Framingham Heart Study reported an inverse association between plasma NT-proBNP levels and intra-abdominal visceral fat accumulation, even after adjusting for BMI, although the association was not significant with subcutaneous fat. ${ }^{13}$ Similarly, the Dallas Heart Study reported a significant association between higher NT-proBNP levels and lower intra-hepatic fat content, as measured by magnetic resonance spectroscopy. ${ }^{14}$ Moreover, higher plasma NT-proBNP levels were also associated with lower prevalence of diabetes and metabolic syndrome. ${ }^{14}$ All these findings suggest that higher NT-proBNP levels might exert protective effects against many CVD risk factors. In line with this, due to the increasing evidence showing that the presence and severity of NAFLD is strongly associated with an increased risk of incident CVD events, ${ }^{2}$ NT-proBNP might also be a viable target for future therapeutic investigation for NAFLD-related CVD. 
To date, it is not yet completely understood why an inverse association exists between plasma NT-proBNP levels and NASH. In particular, it remains unclear why in our study we found that lower circulating levels of NT-proBNP were significantly associated with greater histologic grade of steatosis and hepatic ballooning, but not with lobular inflammation. That said, Sonoyama et al. showed that BNP attenuated the activation of hepatic stellate cells and reduced hepatocyte damage resulting from carbon tetrachloride in a mouse model. ${ }^{15}$ This suggests that BNP is associated with reduced overall intra-hepatic inflammation in NASH. Other experimental studies have also shown that NP-receptors expressed in adipose tissue can stimulate lipolysis and the 'browning' of adipocytes, leading to inhibition of visceral ectopic fat accumulation. ${ }^{16}$ Furthermore, a number of studies have demonstrated that BNP plays an important role in stimulating lipid oxidation, fighting against visceral lipid synthesis, which may conceivably exert an ameliorating effect on liver fat accumulation in patients with $\mathrm{NASH} .{ }^{7,17}$ The role of stimulating lipid mobilization and enhancing energy expenditure of NPs were also examined previously. When various NPs (i.e. BNP and atrial NP) are activated, cyclic guanosine monophosphate levels are increased and lead to protein kinase $\mathrm{G}$ activation, resulting in phosphorylation and physical alteration of lipid droplets facilitating adipose triglyceride lipase and energy production. ${ }^{18,19}$ Miyashita et al reported that in transgenic mice overexpressing BNP, high-fat diet induced higher expression of mitochondrial oxidative activities in the skeletal muscle. ${ }^{20}$ In addition, Engeli et al reported that myotubes significantly increased in response to increased BNP levels which led to higher mitochondrial fat oxidative capacity in cultured human skeletal muscles, ${ }^{21}$ indicating that higher levels of NP are associated with a better lipolytic effect and energy expenditure in skeletal and fatty tissues. NP signaling might also influence body fat distribution differently between organs in humans in that the extent of lipolysis may vary among 
different tissues, thus resulting in different levels of fatty deposits. This is evident as the rate of lipolysis is the highest in visceral fat and the lowest in subcutaneous gluteal-femoral fat. ${ }^{22}$ However, whether NT-proBNP preferentially influences lipolysis in the liver is not explained in our investigation. Taken together, it is conceivable that higher levels of NPs would stimulate better lipolysis and energy expenditure in the skeletal muscle and adipose tissue, via increased expression of mitochondrial oxidative genes, which could explain the inverse association we observed between plasma NT-proBNP levels and presence of NASH.

\section{Strengths and Limitations}

The main strength of our study was that all participants underwent liver biopsy, which is the reference method for diagnosing and staging NAFLD. ${ }^{23}$ The main limitations were the crosssectional design of the study, which does not allow to draw any definite conclusion on temporal and causal relationships between NASH and circulating NT-proBNP levels. Therefore, the findings of this study should be regarded as hypothesis-generating, and will require further validation in future prospective studies. Secondly, individuals with plasma NT-proBNP levels above the upper limit of normal were excluded, (although all subjects did not have a prior history of CVD), therefore making it unlikely that our results are a type I error, since exclusion of high levels of NT-BNP would tend to bias our results towards the null. Thirdly, the enrollment of only individuals of Chinese Han ethnicity can preclude the extrapolation of these results to other ethnic groups.

\section{Conclusions}


This study showed for the first time that lower plasma NT-proBNP levels are significantly associated with higher prevalence of NASH and histologic NAS score in middle-aged Chinese individuals with biopsy-confirmed NAFLD without a prior history of CVD. Notably, this association remained significant even after adjustment for age, sex, HOMA-estimated insulin resistance, pre-existing diabetes, dyslipidemia and hypertension.

\section{Acknowledgments}

None.

\section{Conflict of interest declaration}

All authors do not have conflicts of interest to disclose.

\section{Financial grants and other funding:}

This work was supported by grants from the National Natural Science Foundation of China (81500665), High Level Creative Talents from Department of Public Health in Zhejiang Province, Project of New Century 551 Talent Nurturing in Wenzhou. GT is supported in part by grants from the University School of Medicine of Verona, Verona, Italy. CDB is supported in part by the Southampton NIHR Biomedical Research Centre (IS-BRC-20004), UK.

Informed consent: Informed consent was obtained from all participants in the study.

Ethics approval statement: Ethical approval for the study was obtained from the ethics committee of the First Affiliated Hospital of Wenzhou Medical University. All procedures 
performed in studies involving human participants were in accordance with the ethical standards of the institutional research committee and with the ethical guidelines of the 1975 Declaration of Helsinki.

\section{REFERENCES}

1. Targher G, Byrne CD, Lonardo A, Zoppini G, Barbui C. Non-alcoholic fatty liver disease and risk of incident cardiovascular disease: A meta-analysis. Journal of hepatology. 2016;65(3):589-600.

2. Byrne CD, Targher G. NAFLD: a multisystem disease. Journal of hepatology. 2015;62(1 Suppl):S47-64.

3. Pemberton CJ, Johnson ML, Yandle TG, Espiner EA. Deconvolution analysis of cardiac natriuretic peptides during acute volume overload. Hypertension. 2000;36(3):355-359.

4. Morgenthaler NG, Struck J, Thomas B, Bergmann A. Immunoluminometric assay for the midregion of pro-atrial natriuretic peptide in human plasma. Clin Chem. 2004;50(1):234236.

5. Sanchez OA, Lazo-Elizondo M, Zeb I, et al. Computerized tomography measured liver fat is associated with low levels of N-terminal pro-brain natriuretic protein (NT-proBNP). Multi-Ethnic Study of Atherosclerosis. Metabolism. 2016;65(5):728-735.

6. Johansen ML, Schou M, Rasmussen J, et al. Low N-terminal pro-brain natriuretic peptide levels are associated with non-alcoholic fatty liver disease in patients with type 2 diabetes. Diabetes Metab. 2019;45(5):429-435.

7. Verboven K, Hansen D, Jocken JWE, Blaak EE. Natriuretic peptides in the control of lipid metabolism and insulin sensitivity. Obes Rev. 2017;18(11):1243-1259.

8. Liu WY, Zheng KI, Pan XY, et al. Effect of PNPLA3 polymorphism on diagnostic performance of various noninvasive markers for diagnosing and staging nonalcoholic fatty liver disease. J Gastroenterol Hepatol. 2019.

9. Zheng KI, Liu WY, Pan XY, et al. Combined and sequential non-invasive approach to diagnosing non-alcoholic steatohepatitis in patients with non-alcoholic fatty liver disease and persistently normal alanine aminotransferase levels. BMJ Open Diabetes Res Care. 2020;8(1).

10. Matthews DR, Hosker JP, Rudenski AS, Naylor BA, Treacher DF, Turner RC. Homeostasis model assessment: insulin resistance and beta-cell function from fasting plasma glucose and insulin concentrations in man. Diabetologia. 1985;28(7):412-419.

11. Sun DQ, Zheng KI, Xu G, et al. PNPLA3 rs 738409 is associated with renal glomerular and tubular injury in NAFLD patients with persistently normal ALT levels. Liver Int. 2020;40(1):107-119.

12. Kleiner DE, Brunt EM, Van Natta M, et al. Design and validation of a histological scoring system for nonalcoholic fatty liver disease. Hepatology (Baltimore, $M d$ ). 2005;41(6):1313-1321.

13. Cheng S, Fox CS, Larson MG, et al. Relation of visceral adiposity to circulating natriuretic peptides in ambulatory individuals. Am J Cardiol. 2011;108(7):979-984. 
14. Neeland IJ, Winders BR, Ayers CR, et al. Higher natriuretic peptide levels associate with a favorable adipose tissue distribution profile. J Am Coll Cardiol. 2013;62(8):752-760.

15. Sonoyama T, Tamura N, Miyashita K, et al. Inhibition of hepatic damage and liver fibrosis by brain natriuretic peptide. FEBS Lett. 2009;583(12):2067-2070.

16. Moro C, Lafontan M. Natriuretic peptides and cGMP signaling control of energy homeostasis. Am J Physiol Heart Circ Physiol. 2013;304(3):H358-368.

17. Santhekadur PK, Kumar DP, Seneshaw M, Mirshahi F, Sanyal AJ. The multifaceted role of natriuretic peptides in metabolic syndrome. Biomed Pharmacother. 2017;92:826-835.

18. Galitzky J, Sengenes C, Thalamas C, et al. The lipid-mobilizing effect of atrial natriuretic peptide is unrelated to sympathetic nervous system activation or obesity in young men. $J$ Lipid Res. 2001;42(4):536-544.

19. Birkenfeld AL, Budziarek P, Boschmann M, et al. Atrial natriuretic peptide induces postprandial lipid oxidation in humans. Diabetes. 2008;57(12):3199-3204.

20. Miyashita K, Itoh H, Tsujimoto H, et al. Natriuretic peptides/cGMP/cGMP-dependent protein kinase cascades promote muscle mitochondrial biogenesis and prevent obesity. Diabetes. 2009;58(12):2880-2892.

21. Engeli S, Birkenfeld AL, Badin PM, et al. Natriuretic peptides enhance the oxidative capacity of human skeletal muscle. J Clin Invest. 2012;122(12):4675-4679.

22. Arner P. Differences in lipolysis between human subcutaneous and omental adipose tissues. Ann Med. 1995;27(4):435-438.

23. Byrne CD, Patel J, Scorletti E, Targher G. Tests for diagnosing and monitoring nonalcoholic fatty liver disease in adults. BMJ. 2018;362:k2734. 


\section{Table legends}

Table 1. Baseline characteristics of patients with biopsy-proven NAFLD, stratified by plasma NT-proBNP tertiles.

Table 2. Association between lower plasma NT-proBNP levels and presence of NASH. 


\section{Figure legends}

Figure 1. Boxplot of plasma NT-proBNP levels in relation to total NAS score and presence of $\mathrm{NASH}$. The central rectangle spans the $1^{\text {st }}$ quartile to the $3^{\text {rd }}$ quartile (i.e., the inter-quartile range $[\mathrm{IQR}])$. The segment inside the rectangle shows the median and the "whiskers" above and below the box show the locations of $1.5 \mathrm{x}$ IQR values. 